\title{
Efficient synthesis of 2,4,5-triaryl substituted imidazoles under solvent free conditions at room temperature
}

\author{
Arshia Parveen, ${ }^{a}$ Md. Rafi Sk. Ahmed, ${ }^{\text {Kabeer A. Shaikh, }{ }^{c} \text { Sudhir P. Deshmukh, }}$ \\ and Rajendra P. Pawar ${ }^{* a}$ \\ ${ }^{a}$ Organic Chemistry Synthesis Laboratory, Dnyanopasak College, Parbhani-431401, MS, India \\ ${ }^{b}$ Department of Zoology, Science College, Nanded-431 602, MS, India \\ ${ }^{c}$ Department of Chemistry, Sir Syyed College, Aurangabad-431001, MS, India \\ E-mail:rppawar@yahoo.com
}

\begin{abstract}
A range of 2,4,5-triaryl substituted imidazoles have been synthesized in very good yields under solvent-free conditions by grinding 1,2-diketones, aromatic aldehydes and ammonium acetate in the presence of molecular iodine as catalyst. The short reaction time, cleaner reaction, and easy workup make this protocol practical and economically attractive.
\end{abstract}

Keywords: Molecular iodine, ammonium acetate, aromatic aldehyde, 2,4,5-triaryl substituted imidazoles, grinding

\section{Introduction}

To design and conduct chemical reactions with "green" experimental protocol is an enormous challenge that chemists have to confront to improve the quality of the environment for present and future generations. Target areas for achieving this goal are the exploration of alternative reaction conditions and reaction media to accomplish the desired chemical transformations with minimized by-products or waste, and elimination of the use of conventional organic solvents, wherever possible. Traditional chemical syntheses or transformations generally require volatile and often hazardous organic solvents as reaction media to facilitate mass and heat transfer, and to isolate and purify desired product from reaction mixtures. Over the past several years, chemists have been aware of the environmental implications of their chemistry. Nowadays, they are trying to develop new synthetic methods, reaction conditions, and uses of chemicals that reduce risks to humans and the environment. Organic solvents are high on the list of damaging chemicals because they are employed in huge amounts and are usually volatile liquids that are difficult to store. 
In recent years, solid-state organic reactions have caused great interest. They have many advantages such as high efficiency and selectivity, easy separation and purification, and mild reaction conditions and benefit industry as well as the environment ${ }^{1}$. Many articles about solidstate reactions with grinding have been reported, such as the Grignard reaction, ${ }^{2}$ aldol condensations, ${ }^{3}$ and other reactions. ${ }^{4}$

Compounds with an imidazole ring system have many pharmacological properties and play important roles in biochemical processes. ${ }^{5}$ Many of the substituted imidazoles are known as inhibitors of fungicides and herbicides, plant growth regulators and therapeutic agents. ${ }^{6}$ Recent advances in green chemistry and organometallic chemistry have extended the boundary of imidazoles to the synthesis and application of a large class of imidazoles as ionic liquids and imidazole related N-heterocyclic carbenes. ${ }^{7}$

There are several methods reported in the literature for the synthesis of imidazoles such as the hetero-Cope rearrangement, ${ }^{8}$ four-component condensation of arylglyoxals, primary amines, carboxylic acids and isocyanides on Wang resin, ${ }^{9}$ reaction of $\mathrm{N}$-(2-oxo)-amides with ammonium trifluoroacetate, 1,2-aminoalcohols in the presence of $\mathrm{PCl}_{5}$, diketones, aldehyde, amine and ammonium acetate in phosphoric acid and in acetic acid, organo catalyst in acetic acid as well as $\mathrm{H}_{2} \mathrm{SO}_{4}$ and DMSO. Several microwave (MW) assisted syntheses of imidazoles from 1,2diketones and aldehydes in the presence of a variety of catalysts such as silica-gel, silica-gel/H$\mathrm{Y}, \mathrm{Al}_{2} \mathrm{O}_{3}$, DMF, acetic acid, ${ }^{10} \mathrm{ZrCl}_{4},{ }^{11} \mathrm{NiCl}_{2} \cdot 6 \mathrm{H}_{2} \mathrm{O},{ }^{12}$ and ionic liquid ${ }^{13}$ has been reported.

Many of the synthetic protocols for imidazoles reported so far suffer from one or more disadvantages such as harsh reaction conditions, poor yields, prolonged time period, use of hazardous and often expensive acid catalysts. Moreover, the synthesis of these heterocycles has been usually carried out in polar solvents such as ethanol, methanol, acetic acid, DMF and DMSO leading to complex isolation and recovery procedures. These processes also generate waste containing catalyst and solvent, which have to be recovered, treated and disposed off.

Recently, molecular iodine has received considerable attention as an inexpensive, nontoxic, readily available catalyst for various organic transformations, affording the corresponding products in excellent yields with high selectivity. The mild lewis acidity associated with iodine enhanced its usage in organic synthesis to realize several organic transformations using stoichiometric levels to catalytic amounts. Owing to numerous advantages associated with this eco-friendly element, iodine has been explored as a powerful catalyst for various organic transformations. ${ }^{14}$ During the course of our studies towards the development of new routes to the synthesis of biologically active heterocycles we wish to report a simple and efficient method for the synthesis of 2,4,5-triaryl substituted imidazoles. 


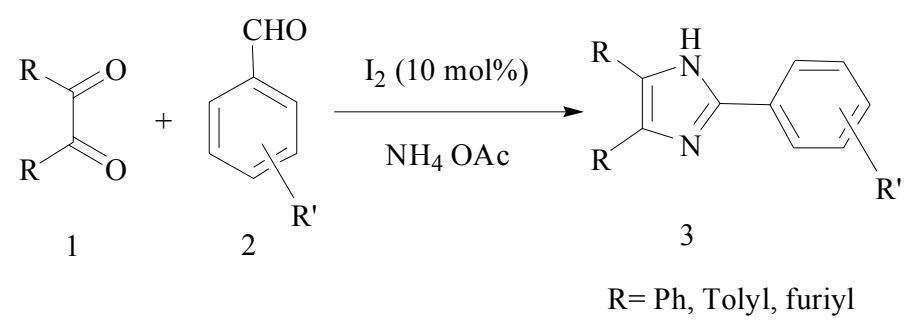

\section{Scheme 1}

\section{Results and Discussion}

Reactions were carried out simply by mixing 1,2-diketone with an aldehyde and ammonium acetate in the presence of a catalytic amount $(15 \mathrm{~mol} \%)$ of iodine under solvent-free condition. The mixture was ground together in a mortar with a pestle at room temperature for several minutes, and then purified by column chromatography, 2,4,5-triaryl substituted imidazole derivatives were obtained in excellent yields. Accordingly, $10 \mathrm{~mol} \%$ was sufficient to catalyze the reaction. A rate enhancement with high yield was observed when higher molar ratios of iodine were used. However, no product formation was observed in absence of iodine.

Table 1. Catalytic evolution for synthesis of 3a under solvent free conditions

\begin{tabular}{cccc}
\hline Entry & $\mathrm{I}_{2}(\mathrm{~mol} \mathrm{\% )}$ & $\begin{array}{c}\text { Time } \\
(\mathrm{min} .)\end{array}$ & $\begin{array}{c}\text { Yield }^{\mathrm{a}} \\
(\%)\end{array}$ \\
\hline 1 & No iodine & 180 & 00 \\
2 & 1 & 60 & traces \\
3 & 2 & 60 & 20 \\
4 & 5 & 60 & 85 \\
5 & 10 & 15 & 90 \\
6 & 15 & 10 & 90 \\
\hline
\end{tabular}

${ }^{\mathrm{a}}$ Isolated yield after column chromatography.

By getting this result, we have extended this protocol to a variety of aldehydes and diketones summarized in Table 2. This protocol is rapid and efficient for the preparation of several 2,4,5triaryl substituted imidazoles from both electrons efficient as well as electron deficient aromatic aldehydes. Electron-withdrawing groups enhance the rate of the reaction as compare to the electron-donating group. Aliphatic aldehyde and ketones (e.g. acetaldehyde, acetone) were also used as starting carbonyl compounds for the same reaction. No product formation takes place in this reaction by grinding the reagents for more than 30 minutes. The aryl groups substituted with different groups did not show any effect on the formation of imidazoles. The ortho and para substituents activate the aromatic ring of aldehydes and increase the rate of the reaction. While 
meta substitution requires somewhat greater time as compared to the $o / p$ substituents. Heteroaromatic diketones reacted fast and gave excellent yields of desired imidazoles. A nearly stiochiometric amount of ammonium acetate was used in the course of the reaction, whereas previously a many-fold excess of ammonium acetate was required. This is an additional advantage of the novel methodology. The possible mechanism of this reaction is as follows:
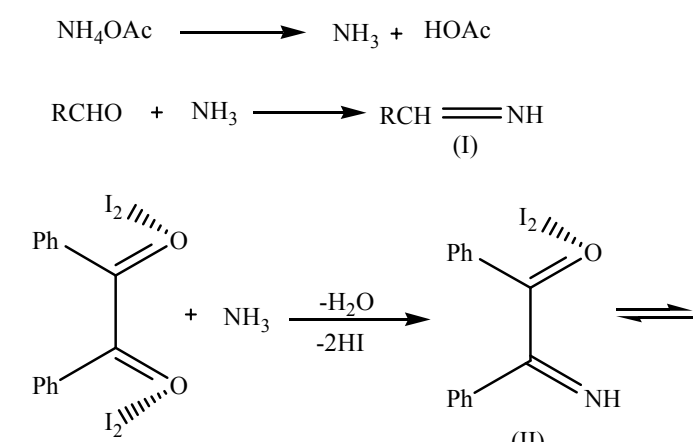

(II)

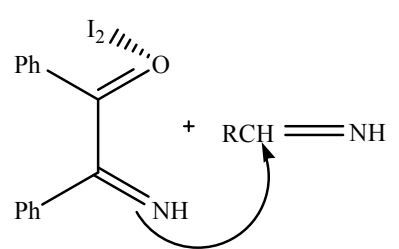

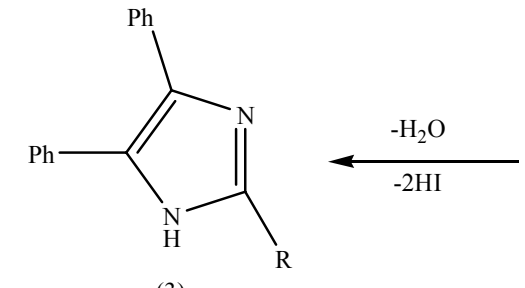

(3)

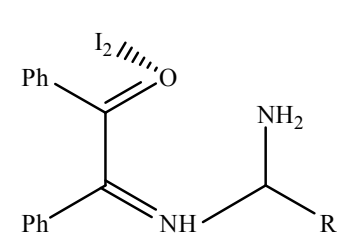

(III)

\section{Scheme 2}

The Lewis acidic nature of molecular iodine makes it capable of binding with the carbonyl oxygen of aromatic aldehyde increasing the reactivity of the parent carbonyl compound and facilitates the formation of imines intermediate (I). Further catalyst iodine condenses with the carbonyl oxygens of the 1,2-diketone, which on dehydration afford the intermediate (II). Intermediates I and II combine for the formation of intermediate III, which on dehydration and further cyclisation gives 2,4,5 triaryl substituted imidazoles (3) Scheme 2.

All the above observations indicate the limited scope of this method, which is only applicable efficiently for aromatic aldehydes. 
Table 2. Synthesis of 2,4,5-trisubstuited imidazoles using (15 mol\%) molecular iodine

\begin{tabular}{|c|c|c|c|c|}
\hline \multirow[t]{2}{*}{ Entry } & & & \multirow[t]{2}{*}{$\begin{array}{l}\text { Time } \\
\text { (min.) }\end{array}$} & \multirow[t]{2}{*}{$\begin{array}{c}\text { Yield }^{\mathrm{a}} \\
(\%)\end{array}$} \\
\hline & $\mathrm{R}$ & $\mathrm{R}^{\prime}$ & & \\
\hline $3 a$ & $\mathrm{Ph}$ & H & 10 & 90 \\
\hline $3 b$ & $\mathrm{Ph}$ & $p$-OMe & 15 & 85 \\
\hline $3 \mathrm{c}$ & $\mathrm{Ph}$ & $m$-OMe, $p-\mathrm{OH}$ & 15 & 87 \\
\hline $3 \mathrm{~d}$ & Toluene & $\mathrm{H}$ & 10 & 82 \\
\hline $3 e$ & Toluene & $p$-OMe & 14 & 85 \\
\hline $3 f$ & Toluene & $p-\mathrm{Br}$ & 18 & 90 \\
\hline $3 \mathrm{~g}$ & Toluene & $\mathrm{m}-\mathrm{OH}$ & 20 & 83 \\
\hline $3 \mathrm{~h}$ & Furan & $\mathrm{H}$ & 10 & 90 \\
\hline $3 \mathrm{i}$ & Furan & $p$-OMe & 12 & 90 \\
\hline
\end{tabular}

${ }^{a}$ Isolated yield after column chromatography

In conclusion, molecular iodine was found to be a mild and effective catalyst for the formation of 2,4,5-triaryl substituted imidazoles in excellent yields. The uses of this inexpensive and easily available catalyst under solvent-free conditions make this protocol practical and economically attractive. The simple work-up procedure, mild reaction conditions, selectivity, and very good yields make our methodology a valid contribution to the existing processes in the field of 2,4,5-triaryl substituted imidazole derivatives synthesis.

\section{Experimental Section}

General Procedures. A mixture of benzaldehyde $(1 \mathrm{mmol})$, benzil $(1 \mathrm{mmol}), \mathrm{NH}_{4} \mathrm{OAc}(2.5$ $\mathrm{mmol})$, and iodine $(15 \mathrm{~mol} \%)$ were ground together in a mortar with a pestle at room temperature for appropriate time (Table 2). After completion of reaction confirmed by TLC, the mixture was treated with aqueous $\mathrm{Na}_{2} \mathrm{~S}_{2} \mathrm{O}_{3}$ to furnish the crude products. The crude was further purified by column chromatography by using petroleum ether: ethyl acetate $(9: 1)$ eluent and recrystallised from ethanol.

2,4,5-Triphenyl-1H-imidazole (3a). Pale yellow solid mp. 271-272 ${ }^{\circ} \mathrm{C}$ (Lit. ${ }^{[13]} 272-273{ }^{0} \mathrm{C}$ ). (Found $\mathrm{C}, 85.02, \mathrm{H}, 5.1, \mathrm{~N}, 9.12 \% . \mathrm{C}_{21} \mathrm{H}_{16} \mathrm{~N}_{2}$ requires $\mathrm{C}, 85.11, \mathrm{H}, 5.44, \mathrm{~N}, 9.45 \%$ ) $v_{\max }$ $(\mathrm{KBr}) / \mathrm{cm}^{-1} 3434,2993,2470,1638$ 1216. ${ }^{1} \mathrm{H}$ NMR (CDCl $/$ DMSO-d 6 ): $\delta_{\mathrm{H}} 12.61$ (1H, brs), $7.42-$ $8.12(15 \mathrm{H}, \mathrm{m}) .{ }^{13} \mathrm{C} \mathrm{NMR}\left(\mathrm{CDCl}_{3} / \mathrm{DMSO}_{-} \mathrm{d}_{6}\right): \delta_{\mathrm{C}} 136.5,129.1,128.5 .127 .2,122.1$. 
2-(4-Methoxy-phenyl)-4,5-diphenyl-1H-imidazole (3b). Solid mp $225{ }^{0} \mathrm{C}$; (Lit. ${ }^{[13]} 228{ }^{0} \mathrm{C}$ ). (Found C, 80.68, H, 5.23, N, 8.42\%. $\mathrm{C}_{22} \mathrm{H}_{18} \mathrm{~N}_{2} \mathrm{O}$ requires $\mathrm{C}, 80.96, \mathrm{H}, 5.56, \mathrm{~N}, 8.58 \%$ ) $v_{\max }$ $(\mathrm{KBr}) / \mathrm{cm}^{-1} 3428,2893,2465,1636,1216 .{ }^{1} \mathrm{H}$ NMR $\left(\mathrm{CDCl}_{3} / \mathrm{DMSO}_{-} \mathrm{d}_{6}\right): \delta_{\mathrm{H}} 3.85(\mathrm{~s}, 3 \mathrm{H}), 12.52$ (1H, brs), 8.02-8.05 (2H, d), 7.25-7.59 (10H, m), 6.93-6.96 (2H, d), $3.85(3 \mathrm{H}, \mathrm{s}) .{ }^{13} \mathrm{C}$ NMR $\left(\mathrm{CDCl}_{3} / \mathrm{DMSO}_{\mathrm{d}}\right): \delta_{\mathrm{C}} 159.1,145.7,132.8,127.6,126.5,126.3,122.7,113.2,54.6$.

2-(4,5-Diphenyl-1H-imidazol-2-yl)-3-methoxy phenol (3c). Solid mp. $168{ }^{0} \mathrm{C}\left(\right.$ Lit. $^{[13]}$ ) (Found $\mathrm{C}, 77.05, \mathrm{H}, 5.18, \mathrm{~N}, 8.42 \% . \mathrm{C}_{22} \mathrm{H}_{18} \mathrm{~N}_{2} \mathrm{O}_{2}$ requires $\left.\mathrm{C}, 77.17, \mathrm{H}, 5.30, \mathrm{~N}, 8.18 \%\right) v_{\max }(\mathrm{KBr}) / \mathrm{cm}^{-1}$ 3611, 3412, 2923, 1652, 1253,; ${ }^{1} \mathrm{H}$ NMR $\left(\mathrm{CDCl}_{3} / \mathrm{DMSO}_{-} \mathrm{d}_{6}\right): \delta 3.86$ (s, 3H), 6.82-6.85 (m, 3H), 7.29-7.32 (m, 5H), 7.53-7.55 (m, 5H), 12.5 (brs, $1 \mathrm{H}) ;{ }^{13} \mathrm{C} \mathrm{NMR}\left(\mathrm{CDCl}_{3} / \mathrm{DMSO}_{\mathrm{d}}\right) \delta 126.3$, 126.7, 127.3, 127.4, 129.8, 147.3, 146.1, 145.3, 129.8, 127.4, 127.3, 126.7, 126.3, 117.1, $155.6,, 112.1,110.9,, 54.7$

2-Phenyl-4, 5-di-p-tolyl-1H-imidazole (3d). Mp. $254{ }^{0} \mathrm{C}$; (Lit. ${ }^{[13]}$ ) (Found C, 85.1, H, 6.21, N, $8.50 \% . \mathrm{C}_{23} \mathrm{H}_{20} \mathrm{~N}_{2}$ requires C, 85.18, H, 6.11, N, 8.63\%) $v_{\max }(\mathrm{KBr}) / \mathrm{cm}^{-1} \quad \mathrm{IR}\left(\mathrm{cm}^{-1}\right) 3432,2998$, 2465, 1638, 1216, ; ${ }^{1} \mathrm{H}$ NMR $\left(\mathrm{CDCl}_{3} / \mathrm{DMSO}_{6}\right) \delta 2.36$ (s, 6H), 7.14-7.18 (m, 8H), 7.34-7.39 (m, 5H), 12.58 (brs, $1 \mathrm{H}) ;{ }^{13} \mathrm{C}$ NMR $\left(\mathrm{CDCl}_{3} / \mathrm{DMSO}_{-} \mathrm{d}_{6}\right) \delta$ 135.1. 128.3, 129.2, 129.1, 127.7, $127.5,127.1,126.6,124.1,19.7$,

2-(4-Methoxy-phenyl)-4,5-di-p-tolyl-1H-imidazole (3e). Mp. $243{ }^{0} \mathrm{C}$; (Lit. ${ }^{[13]}$ ) (Found C, 81.2, $\mathrm{H}, 6.11, \mathrm{~N}, 7.50 \% . \mathrm{C}_{24} \mathrm{H}_{22} \mathrm{~N}_{2} \mathrm{O}$ requires $\left.\mathrm{C}, 81.33, \mathrm{H}, 6.26, \mathrm{~N}, 7.90 \%\right) v_{\max }(\mathrm{KBr}) / \mathrm{cm}^{-1} \mathrm{IR}\left(\mathrm{cm}^{-1}\right)$ ,3430, 2988, 2475,1638, 1216,; ${ }^{1} \mathrm{H}$ NMR $\left(\mathrm{CDCl}_{3} / \mathrm{DMSO}_{\mathrm{d}}\right) \delta 2.37$ (s, 6H), 3.86 (s, 3H), 6.94$6.96(\mathrm{~d}, 2 \mathrm{H}), 7.13-7.15(\mathrm{~m}, 4 \mathrm{H}), 7.46-7.48(\mathrm{~m}, 4 \mathrm{H}), 8.03-8.05(\mathrm{~d}, 2 \mathrm{H}), 12.59$ (brs, $1 \mathrm{H}) ;{ }^{13} \mathrm{C}$ NMR $\left(\mathrm{CDCl}_{3} / \mathrm{DMSO}_{-}\right) \delta 158.6,145.1,135.4,128.1,126.9,126.1,122.6,112.9,54.3,20.2$.

2-(4-Bromo-phenyl)-4,5-di-p-tolyl-1H-imidazole (3f). Mp. $215^{0} \mathrm{C}$; (Lit. ${ }^{[13]}$ ) (Found C, 68.29,

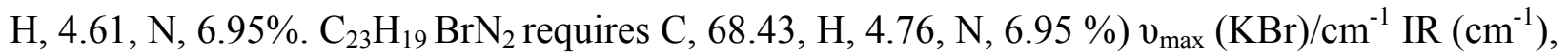
3432, 2978, 2465, 1638,1216; ${ }^{1} \mathrm{H}$ NMR $\left(\mathrm{CDCl}_{3} / \mathrm{DMSO}_{-}\right) \delta 2.35$ (s, 6H), 7.11-7.15 (m, 4H), 7.42-7.46 (m, 4H), 7.54-7.58 (d, 2H), 8.02-8.06 (d, 2H), 12.78 (brs, $1 \mathrm{H}) ;{ }^{13} \mathrm{C} \mathrm{NMR}\left(\mathrm{CDCl}_{3} /\right.$ $\left.\mathrm{DMSO}_{6} \mathrm{~d}_{6}\right) \delta, 142.8,134.7,129.8,127.3,126.1,125.4,119.8,19.4$.

2-(4,5-Di-p-tolyl-1H-imidazol-2-yl)-3-phenol (3g). Mp. $230{ }^{0} \mathrm{C}$; (Lit. ${ }^{[13]}$ ) (Found C, 77.69, H,

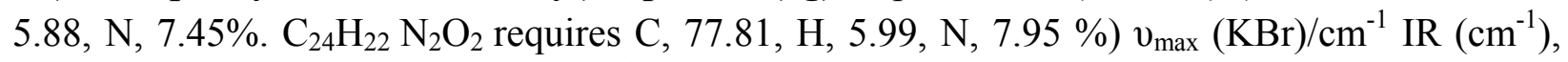
3616, 3442, 2978, 2475, 1638, 1216; ${ }^{1} \mathrm{H}$ NMR $\left(\mathrm{CDCl}_{3} / \mathrm{DMSO}_{-} \mathrm{d}_{6}\right) \delta 2.36(\mathrm{~s}, 6 \mathrm{H}), 6.82-6.85(\mathrm{~m}$, $4 \mathrm{H}), 7.31-7.34(\mathrm{~m}, 4 \mathrm{H}), 7.55-7.58(\mathrm{~m}, 4 \mathrm{H}), 12.71$ (brs, $1 \mathrm{H}) ;{ }^{13} \mathrm{C}$ NMR $\left(\mathrm{CDCl}_{3} / \mathrm{DMSO}-\mathrm{d}_{6}\right) \delta$ $147.3,146.1,129.8,145.1,127.4,127.3,126.7,126.3,117.1,155.6,112.1,110.9$, 54.7, 19.4 .

4,5-Difuran-2-yl-2-phenyl-1H-imidazole (3h). Mp. $218{ }^{0} \mathrm{C}$; (Lit. ${ }^{[13]}$ ) (Found C, 48.29, H, 3.87, $\mathrm{N}, 7.47 \%$. $\mathrm{C}_{17} \mathrm{H}_{11} \mathrm{~N}_{2} \mathrm{O}_{2}$ requires $\left.\mathrm{C}, 48.80, \mathrm{H}, 3.99, \mathrm{~N}, 7.95 \%\right) v_{\max }(\mathrm{KBr}) / \mathrm{cm}^{-1} \mathrm{IR}\left(\mathrm{cm}^{-1}\right), 3058$ ,1602, 1448, 890, 718, ${ }^{1} \mathrm{H}$ NMR $\left(\mathrm{CDCl}_{3} / \mathrm{DMSO}_{6}\right) \delta$ 6.13-6.18 (m, 2H), 6.85-6.89 (d, 2H), 7.18 (s, 2H), 7.22-7.48 (m, 5H), 12.42 (brs, $1 \mathrm{H}) ;{ }^{13} \mathrm{C} \mathrm{NMR}\left(\mathrm{CDCl}_{3} / \mathrm{DMSO}_{-} \mathrm{d}_{6}\right) \delta, 146.3,146.1$, $140.2,128.9,127.9,127.7,125.6,123.9,110.7,106.9$.

4,5-Difuran-2-yl-2(4-methoxy-phenyl-1H-imidazole (3i). Mp. $198{ }^{0} \mathrm{C}$; (Lit. ${ }^{[13]}$ ) (Found C, 52.20, H, 3.90, N, 7.68\%. $\mathrm{C}_{18} \mathrm{H}_{13} \mathrm{~N}_{2} \mathrm{O}_{2}$ requires $\left.\mathrm{C}, 52.80, \mathrm{H}, 3.99, \mathrm{~N}, 7.96 \%\right) v_{\max }(\mathrm{KBr}) / \mathrm{cm}^{-1}$ IR $\left(\mathrm{cm}^{-1}\right), 3105,1610,1508,880,708, ;{ }^{1} \mathrm{H}$ NMR $\left(\mathrm{CDCl}_{3} / \mathrm{DMSO}_{-}\right) \delta 3.75(\mathrm{~s}, 3 \mathrm{H}), 6.14-6.19$ 
(m, 2H), 6.83-6.86 (m, 2H), $7.16(\mathrm{~s}, 2 \mathrm{H}), 7.41(\mathrm{~m}, 2 \mathrm{H}), 7.79-7.82(\mathrm{~m}, 2 \mathrm{H}), 12.53$ (brs, $1 \mathrm{H}) ;{ }^{13} \mathrm{C}$ $\mathrm{NMR}\left(\mathrm{CDCl}_{3} / \mathrm{DMSO}_{-} \mathrm{d}_{6}\right) \delta 162.0,154.1,128.0,128.8,142.2,114.6,111.6,105.2,54.5$.

\section{Acknowledgements}

The authors are thankful to Dr. W. N. Jadhav, Dnyanopasak College, Parbhani for valuable guidance.

\section{References}

1. Tanaka, K.; Toda, F. Chem. Rev. 2000, 100, 1025.

2. Toda, F.; Takumi, H.; Yamaguchi, H. Chem. Exp. 1989, 4, 507.

3. Toda, F.; Tanaka, K.; Hamai, K. J. Chem. Soc., Perkin Trans. 1 1990, 3207.

4. (a) Schmeyers, T.; Toda, F.; Boy, J.; Kaupp, G. J. Chem., Soc., Perkin Trans. 2 1998, 989.

(b) Hagiwara, H.; Obtsubo, S.; Kato, M. Mol. Cryst. Liq. Cryst. 1996, 279, 291. (c) Tanaka, M.; Kobayashi, K. J. Chem. Soc., Chem. Coummun. 1998, 1965. (d) Im, J.; Kim, J.; Kim, S.; Hahu, B.; Toda, F. Tetrahedron Lett. 1997, 38, 451.

5. Lambardino, J. G.; Wiseman, E. H. J. Med. Chem. 1974, 17,1182.

6. Maier, T.; Schmierer, R.; Bauer, K.; Bieringer, H.; Buerstell, H.; Sachse, B. US Patent 820335, 1989; Chem. Abstr. 1989, 111, 19494.

7. 7 (a) Welton, T.; Chem. Rev., 1999, 99, 2071; (b) Hermann, W. A.; Kocher, C.; $\quad$ Angew. Chem. Int. Ed. 1997, 36, 2162.

8. Lantos, I.; Zhang, W. Y.; Shiu, X.; Eggleston, D. S. J. Org. Chem. 1993, 58, 7092.

9. Zhang, C.; Moran, E.J.; Woiwade,T.F.; Short, K. M.; Mjalli, A. M.; Tetrahedron Lett. 1996, 37, 751 .

10. Wolkenberg, S. E.; Wisnoski, D. D.; Leister, W. H.; Wang, Y.; Zhao, Z.; Lindsley, C. W. Org. Lett. 2004, 6, 1453 and references citied therein.

11. Sharma, G. V. M. ; Jyothi, Y. ; Lakshmi, P. S. ; Synthetic Commun. 2006, 36, 2991.

12. Heravi, M. M.; Bakhtiari, K.; Oskooie, H. A.; Taheri, S.; J. Mol. Cata. A: Chemical 2007, 263, 279.

13. Siddiqui, S. A.; Narkhede, U. C.; Palimkar, S. S.; Daniel, T.; Lahoti, R. J.; Srinivasan, K. V. Tetrahedron 2005, 61, 3539.

14. Jianwei, S.; Dong, Y.; Cao, L.; Wang, X.; Wang, S.; Hu, Y .Y. J. Org. Chem. 2004, 69, 8932. 